\title{
On generalized weakly symmetric $\alpha$-cosymplectic manifolds
}

\author{
Selahattin Beyendi ${ }^{1}$ (D), Mustafa Yıldırım*2 (1D) \\ ${ }^{1}$ Department of Mathematics, Faculty of Education, Inönü University, 44000, Malatya, Turkey \\ ${ }^{2}$ Department of Mathematics, Faculty of Art and Science, Aksaray University, 68100, Aksaray, Turkey
}

\begin{abstract}
This study is concerned with some results on generalized weakly symmetric and generalized weakly Ricci-symmetric $\alpha$-cosymplectic manifolds. We prove the necessary and sufficient conditions for an $\alpha$-cosymplectic manifold to be generalized weakly symmetric and generalized weakly Ricci-symmetric. On the basis of these results, we give one proper example of generalized weakly symmetric $\alpha$-cosymplectic manifolds.
\end{abstract}

Mathematics Subject Classification (2020). Primary 53C15, 53C25; Secondary 53D10, 53D15

Keywords. weakly symmetric manifold, weakly Ricci-symmetric manifold, generalized weakly symmetric manifold, generalized weakly Ricci-symmetric manifold, $\alpha$-cosymplectic manifold

\section{Introduction}

In 1989, L. Tamassy and T. Q. Binh introduced the notions of weakly symmetric and weakly Ricci-symmetric manifolds [18]. Later on, many researchers have studied this topic. For details, we refer the reader to $[3,5,8,11,12,14,16,17,21]$ and the references there in. In the view of [18], a $(2 n+1)$-dimensional $\alpha$ - cosymplectic manifold is said to be weakly symmetric manifold, if its curvature tensor $\tilde{R}$ of type $(0,4)$ is not identically zero and admits the following identity:

$$
\begin{aligned}
\left(\nabla_{W} \tilde{R}\right)\left(X_{1}, X_{2}, X_{3}, X_{4}\right)= & \mathcal{A}_{1}(W) \tilde{R}\left(X_{1}, X_{2}, X_{3}, X_{4}\right)+\mathcal{B}_{1}\left(X_{1}\right) \tilde{R}\left(W, X_{2}, X_{3}, X_{4}\right) \\
& +\mathcal{B}_{1}\left(X_{2}\right) \tilde{R}\left(X_{1}, W, X_{3}, X_{4}\right)+\mathfrak{D}_{1}\left(X_{3}\right) \tilde{R}\left(X_{1}, X_{2}, W, X_{4}\right)(1.1) \\
& +\mathfrak{D}_{1}\left(X_{4}\right) \tilde{R}\left(X_{1}, X_{2}, X_{3}, W\right),
\end{aligned}
$$

where $\nabla$ denotes the Levi-Civita connection with respect to metric $g$ on $M$, also $\mathcal{A}_{1}, \mathcal{B}_{1}, \mathcal{D}_{1}$ are non-zero 1-forms defined by $\mathcal{A}_{1}(W)=g\left(W, \sigma_{1}\right), \mathcal{B}_{1}(W)=g\left(W, \varrho_{1}\right)$ and $\mathfrak{D}_{1}(W)=$ $g\left(W, \pi_{1}\right)$, for all $W$ and $\tilde{R}\left(X_{1}, X_{2}, X_{3}, X_{4}\right)=g\left(R\left(X_{1}, X_{2}\right) X_{3}, X_{4}\right)$. A $(2 n+1)$-dimensional $\alpha$-cosymplectic manifold of this kind is denoted by a $(W S)_{2 n+1}$-manifold. Dubey [9] presented generalized recurrent manifold. In keeping with this work, we shall describe

*Corresponding Author.

Email addresses: selahattin.beyendi@inonu.edu.tr (S. Beyendi), mustafayldrm24@gmail.com (M. Yıldırım)

Received: 22.01.2020; Accepted: 27.07.2021 
a $(2 n+1)$-dimensional $\alpha$-cosymplectic manifold generalized weakly symmetric (briefly $(G W S)_{2 n+1}$-manifold) if it admits the following equation:

$$
\begin{aligned}
\left(\nabla_{W} \tilde{R}\right)\left(X_{1}, X_{2}, X_{3}, X_{4}\right)= & \mathcal{A}_{1}(W) \tilde{R}\left(X_{1}, X_{2}, X_{3}, X_{4}\right)+\mathcal{B}_{1}\left(X_{1}\right) \tilde{R}\left(W, X_{2}, X_{3}, X_{4}\right) \\
& +\mathcal{B}_{1}\left(X_{2}\right) \tilde{R}\left(X_{1}, W, X_{3}, X_{4}\right)+\mathcal{D}_{1}\left(X_{3}\right) \tilde{R}\left(X_{1}, X_{2}, W, X_{4}\right) \\
& +\mathcal{D}_{1}\left(X_{4}\right) \tilde{R}\left(X_{1}, X_{2}, X_{3}, W\right)+\mathcal{A}_{2}(W) \tilde{G}\left(X_{1}, X_{2}, X_{3}, X_{4}\right) \\
& +\mathcal{B}_{2}\left(X_{1}\right) \tilde{G}\left(W, X_{2}, X_{3}, X_{4}\right)+\mathcal{B}_{2}\left(X_{2}\right) \tilde{G}\left(X_{1}, W, X_{3}, X_{4}\right) \\
& +\mathcal{D}_{2}\left(X_{3}\right) \tilde{G}\left(X_{1}, X_{2}, W, X_{4}\right)+\mathcal{D}_{2}\left(X_{4}\right) \tilde{G}\left(X_{1}, X_{2}, X_{3}, W\right),
\end{aligned}
$$

where

$$
\tilde{G}\left(X_{1}, X_{2}, X_{3}, X_{4}\right)=\left[g\left(X_{2}, X_{3}\right) g\left(X_{1}, X_{4}\right)-g\left(X_{1}, X_{3}\right) g\left(X_{2}, X_{4}\right)\right]
$$

and $\mathcal{A}_{i}, \mathcal{B}_{i}, \mathcal{D}_{i}$ are non-zero 1-forms defined by $\mathcal{A}_{i}(W)=g\left(W, \sigma_{i}\right), \mathcal{B}_{i}(W)=g\left(W, \varrho_{i}\right)$ and $\mathcal{D}_{i}(W)=g\left(W, \pi_{i}\right)$, for $i=1,2$. There are interesting results of such $(G W S)_{2 n+1}$-manifolds in that it exhibits

(i) (for $\mathcal{A}_{i}=\mathcal{B}_{i}=\mathcal{D}_{i}=0$ ), locally symmetric space [6],

(ii) (for $\mathcal{A}_{1} \neq 0, \mathcal{A}_{2}=\mathcal{B}_{i}=\mathcal{D}_{i}=0$ ), recurrent space [20],

(iii) (for $\mathcal{A}_{i} \neq 0, \mathcal{B}_{i}=\mathcal{D}_{i}=0$ ), generalized recurrent space [9],

(iv) (for $\frac{\mathcal{A}_{1}}{2}=\mathcal{B}_{1}=\mathcal{D}_{1}=H_{1} \neq 0, \mathcal{A}_{2}=\mathcal{B}_{2}=\mathcal{D}_{2}=0$ ), pseudo symmetric space [7],

(v) (for $\frac{\mathcal{A}_{i}}{2}=\mathcal{B}_{i}=\mathcal{D}_{i}=H_{i} \neq 0$ ), generalized pseudo symmetric space [3],

(vi)) (for $\mathcal{A}_{i}=\mathcal{B}_{2}=\mathcal{D}_{2}=0, \mathcal{B}_{1}=\mathcal{D}_{1} \neq 0$ ), semi-pseudo symmetric space [19],

(vii) (for $\mathcal{A}_{i}=0, \mathcal{B}_{i}=\mathcal{D}_{i} \neq 0$ ), generalized semi-pseudo symmetric space [3],

(viii) (for $\mathcal{A}_{1}=H_{1}+K_{1}, \mathcal{B}_{1}=\mathcal{D}_{1}=H_{1} \neq 0$ and $\mathcal{A}_{2}=\mathcal{B}_{2}=\mathcal{D}_{2}=0$ ), almost pseudo symmetric space [7],

(ix) (for $\mathcal{A}_{i}=H_{i}+K_{i}, \mathcal{B}_{i}=\mathcal{D}_{i}=H_{i} \neq 0$ ), almost generalized pseudo symmetric space [3],

(x) (for $\mathcal{A}_{1}, \mathcal{B}_{1}, \mathcal{D}_{i} \neq 0, \mathcal{A}_{2}=\mathcal{B}_{2}=\mathcal{D}_{2}=0$ ), weakly symmetric space [18] .

In the present paper, we have investigated some properties of the generalized weakly symmetric $\alpha$-cosymplectic manifolds. In Section 2, we review basic formulas and definitions for $\alpha$-cosymplectic manifolds. In Section 3 , we have examined a generalized weakly symmetric $\alpha$-cosymplectic manifold and it is observed that such a space is an $\eta$-Einstein manifold provided $\mathcal{D}_{1}(\xi) \neq-\alpha$. We also present tables of different types of curvature restrictions for which $\alpha$-cosymplectics manifolds are sometimes Einstein and some other times remain $\eta$-Einstein. In Section 4, we have given an example of the existence of such manifolds. Finally, we have investigated a generalized weakly Ricci-symmetric $\alpha$-cosymplectic manifold which is also found to be $\eta$-Einstein space.

\section{Preliminaries}

Let $\left(M^{2 n+1}, \varphi, \xi, \eta, g\right)$ be a $(2 n+1)$-dimensional almost contact metric manifold, where $\varphi$ is a $(1,1)$-tensor field, $\xi$ is the structure vector field, $\eta$ is a 1 -form and $g$ is the Riemannian metric. It is well known that the $(\varphi, \xi, \eta, g)$ structure satisfies the following conditions [5]:

$$
\varphi(\xi)=0, \quad \eta(\varphi)=0, \quad \eta(\xi)=1,
$$




$$
\begin{gathered}
\varphi^{2} W=-W+\eta(W) \xi, \quad g(W, \xi)=\eta(W), \\
g\left(\varphi W, \varphi X_{1}\right)=g\left(W, X_{1}\right)-\eta(W) \eta\left(X_{1}\right),
\end{gathered}
$$

for any vector fields $W$ and $X_{1}$ on $M^{2 n+1}$. If in addition,

$$
\begin{gathered}
\nabla_{W} \xi=-\alpha \varphi^{2} W \\
\left(\nabla_{W} \eta\right) X_{1}=\alpha\left[g\left(W, X_{1}\right)-\eta(W) \eta\left(X_{1}\right)\right],
\end{gathered}
$$

where $\nabla$ denotes the Riemannian connection holds and $\alpha$ is a real number, then $\left(M^{2 n+1}, \varphi, \xi, \eta, g\right)$ is called an $\alpha$-cosymplectic manifold $([1,2,10,13,15])$. In this case, it is well known that [4]

$$
\begin{gathered}
R\left(W, X_{1}\right) \xi=\alpha^{2}\left[\eta(W) X_{1}-\eta\left(X_{1}\right) W\right], \\
S(W, \xi)=-2 n \alpha^{2} \eta(W), \\
S(\xi, \xi)=-2 n \alpha^{2},
\end{gathered}
$$

where $S$ denotes the Ricci tensor. From (2.6), it easily follows that

$$
\begin{gathered}
R(W, \xi) X_{1}=\alpha^{2}\left[g\left(W, X_{1}\right) \xi-\eta\left(X_{1}\right) W\right], \\
R(W, \xi) \xi=\alpha^{2}[\eta(W) \xi-W],
\end{gathered}
$$

for any vector fields $W, X_{1}$ where $R$ denotes the Riemannian curvature tensor of $M$. An $\alpha$-cosymplectic manifold is said to be an $\eta$-Einstein manifold if the Ricci tensor $S$ satisfies condition

$$
S\left(W, X_{1}\right)=\lambda_{1} g\left(W, X_{1}\right)+\lambda_{2} \eta(W) \eta\left(X_{1}\right),
$$

where $\lambda_{1}, \lambda_{2}$ are certain scalars.

\section{Generalized weakly symmetric $\alpha$-cosymplectic manifold}

In this section, let us consider a generalized weakly symmetric $\alpha$-cosymplectic manifold $\left(M^{2 n+1}, g\right)(n \geq 1)$. Now, contracting $X_{1}$ over $X_{4}$ in both sides of (1.2), we get

$$
\begin{aligned}
\left(\nabla_{W} S\right)\left(X_{2}, X_{3}\right)= & \mathcal{A}_{1}(W) S\left(X_{2}, X_{3}\right)+\mathcal{B}_{1}\left(R\left(W, X_{2}\right) X_{3}\right)+\mathcal{B}_{1}\left(X_{2}\right) S\left(W, X_{3}\right) \\
& +\mathcal{D}_{1}\left(X_{3}\right) S\left(X_{2}, W\right)+\mathcal{D}_{1}\left(R\left(W, X_{3}\right) X_{2}\right)+2 n \mathcal{A}_{2}(W) g\left(X_{2}, X_{3}\right) \\
& +2 n \mathcal{B}_{2}\left(X_{2}\right) g\left(W, X_{3}\right)+2 n \mathcal{D}_{2}\left(X_{3}\right) g\left(W, X_{2}\right) \\
& +\mathcal{B}_{2}(W) g\left(X_{2}, X_{3}\right)-\mathcal{B}_{2}\left(X_{2}\right) g\left(W, X_{3}\right)+\mathcal{D}_{2}(W) g\left(X_{2}, X_{3}\right) \\
& -\mathcal{D}_{2}\left(X_{3}\right) g\left(X_{2}, W\right) .
\end{aligned}
$$

Putting $X_{3}=\xi$ in (3.1), we obtain

$$
\begin{aligned}
\left(\nabla_{W} S\right)\left(X_{2}, \xi\right)= & \mathcal{A}_{1}(W) S\left(X_{2}, \xi\right)+\mathcal{B}_{1}\left(R\left(W, X_{2}\right) \xi\right)+\mathcal{B}_{1}\left(X_{2}\right) S(W, \xi) \\
& +\mathcal{D}_{1}(\xi) S\left(X_{2}, W\right)+\mathcal{D}_{1}\left(R(W, \xi) X_{2}\right)+2 n \mathcal{A}_{2}(W) \eta\left(X_{2}\right) \\
& +2 n \mathcal{B}_{2}\left(X_{2}\right) \eta(W)+2 n \mathcal{D}_{2}(\xi) g\left(X_{2}, W\right)+\mathcal{B}_{2}(W) \eta\left(X_{2}\right) \\
& -\mathcal{B}_{2}\left(X_{2}\right) \eta(W)+\mathcal{D}_{2}(W) \eta\left(X_{2}\right)-\mathcal{D}_{2}(\xi) g\left(X_{2}, W\right) .
\end{aligned}
$$

Using (2.6), (2.7) and (2.9) in (3.2), we get

$$
\begin{aligned}
\left(\nabla_{W} S\right)\left(X_{2}, \xi\right)= & -2 n \alpha^{2} \mathcal{A}_{1}(W) \eta\left(X_{2}\right)+\alpha^{2} \mathcal{B}_{1}\left(X_{2}\right) \eta(W)-\alpha^{2} \mathcal{B}_{1}(W) \eta\left(X_{2}\right) \\
& -2 n \alpha^{2} \mathcal{B}_{1}\left(X_{2}\right) \eta(W)+\mathcal{D}_{1}(\xi) S\left(X_{2}, W\right)+\alpha^{2} \mathcal{D}_{1}(\xi) g\left(X_{2}, W\right) \\
& -\alpha^{2} \mathcal{D}_{1}(W) \eta\left(X_{2}\right)+2 n \mathcal{A}_{2}(W) \eta\left(X_{2}\right)+2 n \mathcal{B}_{2}\left(X_{2}\right) \eta(W) \\
& +2 n \mathcal{D}_{2}(\xi) g\left(X_{2}, W\right)+\mathcal{B}_{2}(W) \eta\left(X_{2}\right)-\mathcal{B}_{2}\left(X_{2}\right) \eta(W) \\
& +\mathcal{D}_{2}(W) \eta\left(X_{2}\right)-\mathcal{D}_{2}(\xi) g\left(X_{2}, W\right) .
\end{aligned}
$$

We know that

$$
\left(\nabla_{W} S\right)\left(X_{2}, X_{3}\right)=\nabla_{W} S\left(X_{2}, X_{3}\right)-S\left(\nabla_{W} X_{2}, X_{3}\right)-S\left(X_{2}, \nabla_{W} X_{3}\right) .
$$

Next we take $X_{3}=\xi$ in (3.4) and then using (2.2), (2.4) and (2.7), we obtain

$$
\left(\nabla_{W} S\right)\left(X_{2}, \xi\right)=-2 n \alpha^{3} g\left(X_{2}, W\right)-\alpha S\left(X_{2}, W\right) .
$$


Now, using (3.5) in (3.3), we have

$$
\begin{aligned}
& -2 n \alpha^{3} g\left(X_{2}, W\right)-\alpha S\left(X_{2}, W\right) \\
& =-2 n \alpha^{2} \mathcal{A}_{1}(W) \eta\left(X_{2}\right)-(2 n-1) \alpha^{2} \mathcal{B}_{1}\left(X_{2}\right) \eta(W)+\mathcal{D}_{1}(\xi) S\left(X_{2}, W\right) \\
& -\alpha^{2} \mathcal{B}_{1}(W) \eta\left(X_{2}\right)-\alpha^{2} \mathcal{D}_{1}(W) \eta\left(X_{2}\right)+\alpha^{2} \mathcal{D}_{1}(\xi) g\left(X_{2}, W\right) \\
& +2 n\left[\mathcal{A}_{2}(W) \eta\left(X_{2}\right)+\mathcal{B}_{2}\left(X_{2}\right) \eta(W)+\mathcal{D}_{2}(\xi) g\left(X_{2}, W\right)\right] \\
& +\mathcal{B}_{2}(W) \eta\left(X_{2}\right)-\mathcal{B}_{2}\left(X_{2}\right) \eta(W)+\mathcal{D}_{2}(W) \eta\left(X_{2}\right)-\mathcal{D}_{2}(\xi) g\left(X_{2}, W\right),
\end{aligned}
$$

which results in

$$
\alpha^{2}\left[\mathcal{A}_{1}(\xi)+\mathcal{B}_{1}(\xi)+\mathcal{D}_{1}(\xi)\right]=\left[\mathcal{A}_{2}(\xi)+\mathcal{B}_{2}(\xi)+\mathcal{D}_{2}(\xi)\right]
$$

for $W=X_{2}=\xi$. In particular, if $\mathcal{A}_{2}(\xi)=\mathcal{B}_{2}(\xi)=\mathcal{D}_{2}(\xi)=0$, equation (3.7) turns into

$$
\alpha^{2}\left[\mathcal{A}_{1}(\xi)+\mathcal{B}_{1}(\xi)+\mathcal{D}_{1}(\xi)\right]=0 .
$$

Theorem 3.1. In a generalized weakly symmetric $\alpha$-cosymplectic manifold $\left(M^{2 n+1}, g\right)$, the relation (3.7) is hold.

Putting $X_{2}=\xi$ in (3.1) and using (3.4), we obtain

$$
\begin{aligned}
& -2 n \alpha^{3} g\left(W, X_{3}\right)-\alpha S\left(W, X_{3}\right) \\
& =-2 n \alpha^{2} \mathcal{A}_{1}(W) \eta\left(X_{3}\right)+\alpha^{2} g\left(W, X_{3}\right) \mathcal{B}_{1}(\xi)-\alpha^{2} \mathcal{B}_{1}(W) \eta\left(X_{3}\right) \\
& +\mathcal{B}_{1}(\xi) S\left(W, X_{3}\right)-(2 n-1) \alpha^{2} \mathcal{D}_{1}\left(X_{3}\right) \eta(W)-\alpha^{2} \mathcal{D}_{1}(W) \eta\left(X_{3}\right) \\
& +2 n\left[\mathcal{A}_{2}(W) \eta\left(X_{3}\right)+\mathcal{B}_{2}(\xi) g\left(W, X_{3}\right)+\mathcal{D}_{2}\left(X_{3}\right) \eta(W)\right] \\
& +\mathcal{B}_{2}(W) \eta\left(X_{3}\right)-\mathcal{B}_{2}(\xi) g\left(W, X_{3}\right)+\mathcal{D}_{2}(W) \eta\left(X_{3}\right)-\mathcal{D}_{2}\left(X_{3}\right) \eta(W) .
\end{aligned}
$$

Taking $X_{3}=\xi$ in (3.9) and also using (2.2) and (2.7), we get

$$
\begin{aligned}
& \alpha^{2}\left[2 n \mathcal{A}_{1}(W)+\mathcal{B}_{1}(W)+\mathcal{D}_{1}(W)+(2 n-1) \eta(W)\left(\mathcal{B}_{1}(\xi)+\mathcal{D}_{1}(\xi)\right)\right] \\
& =2 n \mathcal{A}_{2}(W)+(2 n-1) \eta(W)\left[\mathcal{B}_{2}(\xi)+\mathcal{D}_{2}(\xi)\right]+\mathcal{B}_{2}(W)+\mathcal{D}_{2}(W) .
\end{aligned}
$$

Now putting $W=\xi$ in (3.9) and using (2.1), (2.2) and (2.7), we obtain

$$
\begin{aligned}
& \alpha^{2}\left[2 n\left(\mathcal{A}_{1}(\xi)+\mathcal{B}_{1}(\xi)\right) \eta\left(X_{3}\right)+(2 n-1) \mathcal{D}_{1}\left(X_{3}\right)+\mathcal{D}_{1}(\xi) \eta\left(X_{3}\right)\right] \\
= & 2 n\left[\left(\mathcal{A}_{2}(\xi)+\mathcal{B}_{2}(\xi)\right) \eta\left(X_{3}\right)+\mathcal{D}_{2}\left(X_{3}\right)\right]+\mathcal{D}_{2}(\xi) \eta\left(X_{3}\right)-\mathcal{D}_{2}\left(X_{3}\right) .
\end{aligned}
$$

Replacing $X_{3}$ by $W$ in (3.11) and using (3.7), we have

$$
\alpha^{2} \mathcal{D}_{1}(W)-\alpha^{2} \mathcal{D}_{1}(\xi) \eta(W)=\mathcal{D}_{2}(W)-\mathcal{D}_{2}(\xi) \eta(W) .
$$

Putting $W=\xi$ in (3.6), we get

$$
\begin{aligned}
& -2 n \alpha^{3} \eta\left(X_{2}\right)+2 n \alpha^{3} \eta\left(X_{2}\right) \\
& =-2 n \alpha^{2} \mathcal{A}_{1}(\xi) \eta\left(X_{2}\right)-(2 n-1) \alpha^{2} \mathcal{B}_{1}\left(X_{2}\right)-2 n \alpha^{2} \mathcal{D}_{1}(\xi) \eta\left(X_{2}\right) \\
& -\alpha^{2} \mathcal{B}_{1}(\xi) \eta\left(X_{2}\right)-\alpha^{2} \mathcal{D}_{1}(\xi) \eta\left(X_{2}\right)+\alpha^{2} \mathcal{D}_{1}(\xi) \eta\left(X_{2}\right) \\
& +2 n\left[\mathcal{A}_{2}(\xi) \eta\left(X_{2}\right)+\mathcal{B}_{2}\left(X_{2}\right)+\mathcal{D}_{2}(\xi) \eta\left(X_{2}\right)\right] \\
& +\mathcal{B}_{2}(\xi) \eta\left(X_{2}\right)-\mathcal{B}_{2}\left(X_{2}\right)+\mathcal{D}_{2}(\xi) \eta\left(X_{2}\right)-\mathcal{D}_{2}(\xi) \eta\left(X_{2}\right) .
\end{aligned}
$$

Replacing $X_{2}$ by $W$ in (3.13) and then using (3.7), (3.12), we obtain

$$
\alpha^{2} \mathcal{B}_{1}(W)-\alpha^{2} \mathcal{B}_{1}(\xi) \eta(W)=\mathcal{B}_{2}(W)-\mathcal{B}_{2}(\xi) \eta(W) .
$$

Using (3.10), (3.12) and (3.14), we get

$$
\alpha^{2}\left[\mathcal{A}_{1}(W)+\left(\mathcal{B}_{1}(\xi)+\mathcal{D}_{1}(\xi)\right) \eta(W)\right]=\mathcal{A}_{2}(W)+\left(\mathcal{B}_{2}(\xi)+\mathcal{D}_{2}(\xi)\right) \eta(W) .
$$

In view of (3.12), (3.14) and (3.15), we obtain

$$
\alpha^{2}\left[\mathcal{A}_{1}(W)+\mathcal{B}_{1}(W)+\mathcal{D}_{1}(W)\right]=\left[\mathcal{A}_{2}(W)+\mathcal{B}_{2}(W)+\mathcal{D}_{2}(W)\right] .
$$

Next, for the choice of $\mathcal{A}_{2}=\mathcal{B}_{2}=\mathcal{D}_{2}=0$, the relation in equation (3.16) yields the following:

$$
\alpha^{2}\left[\mathcal{A}_{1}(W)+\mathcal{B}_{1}(W)+\mathcal{D}_{1}(W)\right]=0 .
$$

This motivates us to state the following theorems. 
Theorem 3.2. Let $\left(M^{2 n+1}, \varphi, \xi, \eta, g\right)$ be a $(G W S)_{2 n+1} \alpha$-cosymplectic manifold, the sum of the associated is given by (3.16).

Theorem 3.3. There does not exist an $\alpha$-cosymplectic manifold which is

(i) recurrent,

(ii) generalized recurrent provided the 1-forms associated to the vector fields are colliner,

(iii) pseudo symmetric,

(iv) generalized semi-pseudo symmetric provided the 1-forms associated to the vector fields are collinear.

Again from (3.6), putting $W=\xi$, we have

$$
\begin{aligned}
& 2 n\left[\alpha^{2} \mathcal{A}_{1}(\xi)-\mathcal{A}_{2}(\xi)+\alpha^{2} \mathcal{D}_{1}(\xi)-\mathcal{D}_{2}(\xi)\right] \eta\left(X_{2}\right) \\
& =\left[-\alpha^{2} \mathcal{B}_{1}(\xi)+\mathcal{B}_{2}(\xi)\right] \eta\left(X_{2}\right)+(2 n-1)\left[-\alpha^{2} \mathcal{B}_{1}\left(X_{2}\right)+\mathcal{B}_{2}\left(X_{2}\right)\right] .
\end{aligned}
$$

Using (3.7), the above equation becomes

$$
\left[\alpha^{2} \mathcal{B}_{1}(\xi)-\mathcal{B}_{2}(\xi)\right] \eta\left(X_{2}\right)=\alpha^{2} \mathcal{B}_{1}\left(X_{2}\right)-\mathcal{B}_{2}\left(X_{2}\right) .
$$

Taking $X_{2}=\xi$ in (3.6) and using (3.7), we obtain

$$
\begin{aligned}
& 2 n\left[\alpha^{2} \mathcal{A}_{1}(W)-\mathcal{A}_{2}(W)\right]+\left[\alpha^{2} \mathcal{B}_{1}(W)-\mathcal{B}_{2}(W)\right]+\left[\alpha^{2} \mathcal{D}_{1}(W)-\mathcal{D}_{2}(W)\right] \\
& =(2 n-1)\left[\alpha^{2} \mathcal{A}_{1}(\xi)-\mathcal{A}_{2}(\xi)\right] \eta(W) .
\end{aligned}
$$

Putting (3.16) in (3.20), we have

$$
\left[\alpha^{2} \mathcal{A}_{1}(\xi)-\mathcal{A}_{2}(\xi)\right] \eta(W)=\alpha^{2} \mathcal{A}_{1}(W)-\mathcal{A}_{2}(W) .
$$

Again from (3.6), we get

$$
\begin{aligned}
& S\left(X_{2}, W\right)= \\
& -\frac{\left[-2 n \alpha^{2} \mathcal{A}_{1}(W)-\alpha^{2} \mathcal{B}_{1}(W)-\alpha^{2} \mathcal{D}_{1}(W)+2 n \mathcal{A}_{2}(W)+\mathcal{B}_{2}(W)+\mathcal{D}_{2}(W)\right]}{\alpha+\mathcal{D}_{1}(\xi)} \eta\left(X_{2}\right) \\
& -\frac{\left[2 n \alpha^{3}+\alpha^{2} \mathcal{D}_{1}(\xi)+2 n \mathcal{D}_{2}(\xi)-\mathcal{D}_{2}(\xi)\right]}{\alpha+\mathcal{D}_{1}(\xi)} g\left(X_{2}, W\right) \\
& +\frac{\left[(2 n-1) \alpha^{2} \mathcal{B}_{1}\left(X_{2}\right)-2 n \mathcal{B}_{2}\left(X_{2}\right)+\mathcal{B}_{2}\left(X_{2}\right)\right]}{\alpha+\mathcal{D}_{1}(\xi)} \eta(W)
\end{aligned}
$$

In view of (3.16), (3.19) and (3.21), we obtain

$$
\begin{aligned}
S\left(X_{2}, W\right)= & -\frac{\left[2 n\left(\alpha^{3}+\mathcal{D}_{2}(\xi)\right)+\alpha^{2} \mathcal{D}_{1}(\xi)-\mathcal{D}_{2}(\xi)\right]}{\alpha+\mathcal{D}_{1}(\xi)} g\left(X_{2}, W\right) \\
& +\frac{(2 n-1)\left[\alpha^{2} \mathcal{A}_{1}(\xi)-\mathcal{A}_{2}(\xi)+\alpha^{2} \mathcal{B}_{1}(\xi)-\mathcal{B}_{2}(\xi)\right]}{\alpha+\mathcal{D}_{1}(\xi)} \eta\left(X_{2}\right) \eta(W) .
\end{aligned}
$$

Theorem 3.4. A generalized weakly symmetric $\alpha$-cosymplectic manifold is an $\eta$-Einstein space provided $\mathcal{D}_{1}(\xi) \neq-\alpha$.

Theorem 3.5. In an $\alpha$-cosymplectic manifold the following table is hold. 


\begin{tabular}{ll}
\hline Type of curvature restriction & $\begin{array}{l}\text { Nature of the space corresponding to } \\
\text { curvature restriction }\end{array}$ \\
\hline locally symmetric space & Einstein space \\
\hline locally recurrent space & $\eta$ - Einstein space \\
\hline generalized recurrent space & $\eta$ - Einstein space \\
\hline pseudo symmetric space & $\eta$ - Einstein space \\
\hline generalized pseudo symmetric space & $\eta$ - Einstein space \\
\hline semi-pseudo symmetric space & $\eta$ - Einstein space \\
\hline generalized semi-pseudo symmetric space & $\eta$ - Einstein space \\
\hline almost pseudo symmetric space & $\eta$ - Einstein space \\
\hline almost generalized pseudo symmetric space & $\eta$ - Einstein space \\
\hline weakly symmetric space & $\eta$-Einstein space \\
\hline
\end{tabular}

\section{Existence of Generalized weakly symmetric $\alpha$-cosymplectic manifold}

Let $M^{3}=\left\{(x, y, z) \in R^{3}\right\}$ be a 3 -dimensional manifold, where $(x, y, z)$ are the standard coordinates in $R^{3}$. The vector fields are

$$
\begin{aligned}
& e_{1}=e^{-2 z} \frac{\partial}{\partial x}, \\
& e_{2}=e^{-2 z} \frac{\partial}{\partial y}, \\
& e_{3}=\frac{\partial}{\partial z} .
\end{aligned}
$$

It is obvious that $\left\{e_{1}, e_{2}, e_{3}\right\}$ are linearly independent at each point of $M^{3}$. Let $g$ be the Riemannian metric defined by

$$
g\left(e_{1}, e_{1}\right)=g\left(e_{2}, e_{2}\right)=g\left(e_{3}, e_{3}\right)=1, \quad g\left(e_{1}, e_{2}\right)=g\left(e_{1}, e_{3}\right)=g\left(e_{2}, e_{3}\right)=0,
$$

and given by the tensor product

$$
\left.g=\frac{1}{e^{-4 z}}(d x \otimes d x+d y \otimes d y)+d z \otimes d z\right) .
$$

Let $\eta$ be the 1-form defined by $\eta(W)=g\left(W, e_{3}\right)$ for any vector field $W$ on $M^{3}$ and $\varphi\left(e_{1}\right)=e_{2}, \quad \varphi\left(e_{2}\right)=-e_{1}, \quad \varphi\left(e_{3}\right)=0$. Then using the linearity of $g$ and $\varphi$, we have

$$
\varphi^{2} W=-W+\eta(W) e_{3}, \quad \eta\left(e_{3}\right)=1, \quad g\left(\varphi W, \varphi X_{1}\right)=g\left(W, X_{1}\right)-\eta(W) \eta\left(X_{1}\right),
$$

for any vector fields on $M^{3}$. It remains to prove that $d \Phi=2 \alpha \eta \wedge \Phi$ and the Nijenhuis torsion tensor of $\varphi$ is zero. It follows that $\Phi\left(e_{1}, e_{2}\right)=-1$ and otherwise $\Phi\left(e_{i}, e_{j}\right)=0$ for $i \leq j$. Therefore, the essential non-zero component of $\Phi$ is as follows:

$$
\Phi\left(\frac{\partial}{\partial x}, \frac{\partial}{\partial z}\right)=-\frac{1}{e^{-4 z}}
$$

and hence

$$
\Phi=-\frac{1}{e^{-4 z}} d x \wedge d y
$$

Consequently, the exterior derivative $d \Phi$ is given by

$$
d \Phi=-\frac{4}{e^{-4 z}} d x \wedge d y \wedge d z .
$$

Since $\eta=d z$, by (4.1) and (4.2), we find

$$
d \Phi=4 \eta \wedge \Phi .
$$

Then,

$$
\left[e_{1}, e_{2}\right]=0, \quad\left[e_{1}, e_{3}\right]=2 e_{1}, \quad\left[e_{2}, e_{3}\right]=2 e_{2} .
$$


In conclusion, it can be noted that Nijenhuis torsion of $\varphi$ is zero. Thus, the manifold is a 2-cosymplectic manifold. Using Koszul's formula, we can get the $\nabla$ operator as follows:

$$
\begin{array}{llll}
\nabla_{e_{1}} e_{3} & =2 e_{1}, & \nabla_{e_{1}} e_{2}=0, & \nabla_{e_{1}} e_{1}=-2 e_{3}, \\
\nabla_{e_{2}} e_{3}=2 e_{2}, & \nabla_{e_{2}} e_{2}=-2 e_{3}, & \nabla_{e_{2}} e_{1}=0, \\
\nabla_{e_{3}} e_{3}=0, & \nabla_{e_{3}} e_{2}=0, & \nabla_{e_{3}} e_{1}=0 .
\end{array}
$$

Then using equation (4.3), the non-vanishing components of $\tilde{R}$ (skew-symmetry and up to symmetry) can clearly be seen:

$$
\tilde{R}\left(e_{1}, e_{3}, e_{1}, e_{3}\right)=\tilde{R}\left(e_{2}, e_{3}, e_{2}, e_{3}\right)=4=\tilde{R}\left(e_{1}, e_{2}, e_{1}, e_{2}\right) .
$$

Since $\left\{e_{1}, e_{2}, e_{3}\right\}$ forms a basis, any vector field $W, X_{1}, X_{2}, X_{3} \in \chi(M)$ can be written as

$$
W=\sum_{i=1}^{3} a_{i} e_{i}, \quad X_{1}=\sum_{i=1}^{3} b_{i} e_{i}, \quad X_{2}=\sum_{i=1}^{3} c_{i} e_{i}, \quad X_{3}=\sum_{i=1}^{3} d_{i} e_{i}
$$

and the components can be obtained from the following relations by the symmetry properties,

$$
\begin{aligned}
& \tilde{R}\left(W, X_{1}, X_{2}, X_{3}\right)=T_{1}=\frac{1}{4}\left[\left(a_{1} b_{2}-a_{2} b_{1}\right)\left(c_{1} d_{2}-c_{2} d_{1}\right)\right] \\
& +\left(a_{1} b_{3}-a_{3} b_{1}\right)\left(c_{1} d_{3}-c_{3} d_{1}\right)+\left(a_{2} b_{3}-a_{3} b_{2}\right)\left(c_{2} d_{3}-c_{3} d_{2}\right) \\
& \tilde{R}\left(e_{1}, X_{1}, X_{2}, X_{3}\right)=\lambda_{1}=\frac{1}{4}\left[b_{3}\left(c_{1} d_{3}-c_{3} d_{1}\right)+b_{2}\left(c_{1} d_{2}-c_{2} d_{1}\right)\right] \\
& \tilde{R}\left(e_{2}, X_{1}, X_{2}, X_{3}\right)=\lambda_{2}=\frac{1}{4}\left[b_{3}\left(c_{2} d_{3}-c_{3} d_{2}\right)-b_{1}\left(c_{1} d_{2}-c_{2} d_{1}\right)\right] \\
& \tilde{R}\left(e_{3}, X_{1}, X_{2}, X_{3}\right)=\lambda_{3}=\frac{1}{4}\left[b_{1}\left(c_{3} d_{1}-c_{1} d_{3}\right)+b_{2}\left(c_{3} d_{2}-c_{2} d_{3}\right)\right] \\
& \tilde{R}\left(W, e_{1}, X_{2}, X_{3}\right)=\lambda_{4}=\frac{1}{4}\left[a_{3}\left(c_{1} d_{3}-c_{3} d_{1}\right)+a_{2}\left(c_{1} d_{2}-c_{2} d_{1}\right)\right] \\
& \tilde{R}\left(W, e_{2}, X_{2}, X_{3}\right)=\lambda_{5}=\frac{1}{4}\left[a_{3}\left(c_{2} d_{3}-c_{3} d_{2}\right)+a_{1}\left(c_{2} d_{1}-c_{1} d_{2}\right)\right] \\
& \tilde{R}\left(W, e_{3}, X_{2}, X_{3}\right)=\lambda_{6}=\frac{1}{4}\left[a_{1}\left(c_{3} d_{1}-c_{1} d_{3}\right)+a_{2}\left(c_{3} d_{2}-c_{2} d_{3}\right)\right] \\
& \tilde{R}\left(W, X_{1}, e_{1}, X_{3}\right)=\lambda_{7}=\frac{1}{4}\left[d_{3}\left(a_{1} b_{3}-a_{3} b_{1}\right)+d_{2}\left(a_{1} b_{2}-a_{2} b_{1}\right)\right] \\
& \tilde{R}\left(W, X_{1}, e_{2}, X_{3}\right)=\lambda_{8}=\frac{1}{4}\left[d_{3}\left(a_{2} b_{3}-a_{3} b_{2}\right)+d_{1}\left(a_{2} b_{1}-a_{1} b_{2}\right)\right] \\
& \tilde{R}\left(W, X_{1}, e_{3}, X_{3}\right)=\lambda_{9}=\frac{1}{4}\left[d_{1}\left(a_{3} b_{1}-a_{1} b_{3}\right)+d_{2}\left(a_{3} b_{2}-a_{2} b_{3}\right)\right] \\
& \tilde{R}\left(W, X_{1}, X_{2}, e_{1}\right)=\lambda_{10}=\frac{1}{4}\left[c_{3}\left(a_{1} b_{3}-a_{3} b_{1}\right)+c_{2}\left(a_{1} b_{2}-a_{2} b_{1}\right)\right] \\
& \tilde{R}\left(W, X_{1}, X_{2}, e_{2}\right)=\lambda_{11}=\frac{1}{4}\left[c_{3}\left(a_{2} b_{3}-a_{3} b_{2}\right)+c_{1}\left(a_{2} b_{1}-a_{1} b_{2}\right)\right] \\
& \tilde{R}\left(W, X_{1}, X_{2}, e_{3}\right)=\lambda_{12}=\frac{1}{4}\left[c_{1}\left(a_{3} b_{1}-a_{1} b_{3}\right)+c_{2}\left(a_{3} b_{2}-a_{2} b_{3}\right)\right] \\
& \tilde{G}\left(W, X_{1}, X_{2}, X_{3}\right)=T_{2}=\left(b_{1} c_{1}+b_{2} c_{2}-b_{3} c_{3}\right)\left(a_{1} d_{1}+a_{2} d_{2}-a_{3} d_{3}\right) \\
& -\left(a_{1} c_{1}+a_{2} c_{2}-a_{3} c_{3}\right)\left(b_{1} d_{1}+b_{2} d_{2}-b_{3} d_{3}\right) \text {. }
\end{aligned}
$$


Now, we calculate the components of $\tilde{R}$ which are the non-vanishing covariant derivatives:

$$
\begin{aligned}
\nabla_{e_{1}} \tilde{R}\left(W, X_{1}, X_{2}, X_{3}\right) & =+2 a_{1} \lambda_{3}-2 a_{3} \lambda_{1}+2 b_{1} \lambda_{6}-2 b_{3} \lambda_{4} \\
& +2 c_{1} \lambda_{9}-2 c_{3} \lambda_{7}+2 d_{1} \lambda_{12}-2 d_{1} \lambda_{10} \\
\nabla_{e_{2}} \tilde{R}\left(W, X_{1}, X_{2}, X_{3}\right) & =+2 a_{2} \lambda_{3}-2 a_{3} \lambda_{2}+2 b_{2} \lambda_{6}-2 b_{3} \lambda_{5} \\
& +2 c_{2} \lambda_{9}-2 c_{3} \lambda_{8}+2 d_{2} \lambda_{12}-2 d_{3} \lambda_{11} \\
\nabla_{e_{3}} \tilde{R}\left(W, X_{1}, X_{2}, X_{3}\right) & =0 .
\end{aligned}
$$

Depending on the following choice of the 1-forms

$$
\begin{aligned}
& \mathcal{A}_{1}\left(e_{1}\right)=\frac{2 a_{1} \lambda_{3}-2 a_{3} \lambda_{1}+2 b_{1} \lambda_{6}-2 b_{3} \lambda_{4}}{T_{1}} \\
& \mathcal{A}_{2}\left(e_{1}\right)=\frac{2 c_{1} \lambda_{9}-2 c_{3} \lambda_{7}+2 d_{1} \lambda_{12}-2 d_{1} \lambda_{10}}{T_{2}} \\
& \mathcal{A}_{1}\left(e_{2}\right)=\frac{2 a_{2} \lambda_{3}-2 a_{3} \lambda_{2}+2 b_{2} \lambda_{6}-2 b_{3} \lambda_{5}}{T_{1}} \\
& \mathcal{A}_{2}\left(e_{2}\right)=\frac{2 c_{2} \lambda_{9}-2 c_{3} \lambda_{8}+2 d_{2} \lambda_{12}-2 d_{3} \lambda_{11}}{T_{2}}
\end{aligned}
$$

one can easily verify the following relations that follow

$$
\begin{aligned}
\nabla_{e_{i}} \tilde{R}\left(W, X_{1}, X_{2}, X_{3}\right)= & \mathcal{A}_{1}\left(e_{i}\right) \tilde{R}\left(W, X_{1}, X_{2}, X_{3}\right)+\mathcal{B}_{1}(W) \tilde{R}\left(e_{i}, X_{1}, X_{2}, X_{3}\right) \\
& +\mathcal{B}_{1}\left(X_{1}\right) \tilde{R}\left(W, e_{i}, X_{2}, X_{3}\right)+\mathcal{D}_{1}\left(X_{2}\right) \tilde{R}\left(W, X_{1}, e_{i}, X_{3}\right) \\
& +\mathcal{D}_{1}\left(X_{3}\right) \tilde{R}\left(W, X_{1}, X_{2}, e_{i}\right)+\mathcal{A}_{2}\left(e_{i}\right) \tilde{G}\left(W, X_{1}, X_{2}, X_{3}\right) \\
& +\mathcal{B}_{2}(W) \tilde{G}\left(e_{i}, X_{1}, X_{2}, X_{3}\right)+\mathcal{B}_{2}\left(X_{1}\right) \tilde{G}\left(W, e_{i}, X_{2}, X_{3}\right) \\
& +\mathcal{D}_{2}\left(X_{2}\right) \tilde{G}\left(W, X_{1}, e_{i}, X_{3}\right)+\mathcal{D}_{2}\left(X_{3}\right) \tilde{G}\left(W, X_{1}, X_{2}, e_{i}\right)
\end{aligned}
$$

for $i=1,2,3$. From the above, we can state the following the theorem.

Theorem 4.1. There exists an $\alpha$-cosymplectic manifold $\left(M^{3}, g\right)$ which is a generalized weakly symmetric $\alpha$-cosymplectic manifold.

\section{Generalized weakly Ricci-symmetric $\alpha$-cosymplectic manifold}

Let $\left(M^{2 n+1}, \varphi, \xi, \eta, g\right)$ be an $\alpha$-cosymplectic manifold. If the manifold is generalized weakly Ricci symmetric manifold then there exists 1 -forms $\tilde{\mathcal{A}}_{i}, \tilde{\mathcal{B}}_{i}$ and $\tilde{\mathcal{D}}_{i}$ that satisfy the condition

$$
\begin{aligned}
\left(\nabla_{W} S\right)\left(X_{2}, X_{3}\right) & =\tilde{\mathcal{A}}_{1}(W) S\left(X_{2}, X_{3}\right)+\tilde{\mathcal{B}}_{1}\left(X_{2}\right) S\left(W, X_{3}\right)+\tilde{\mathcal{D}}_{1}\left(X_{3}\right) S\left(X_{2}, W\right) \\
& +\tilde{\mathcal{A}}_{2}(W) g\left(X_{2}, X_{3}\right)+\tilde{\mathcal{B}}_{2}\left(X_{2}\right) g\left(W, X_{3}\right)+\tilde{\mathcal{D}}_{2}\left(X_{3}\right) g\left(X_{2}, W\right) .
\end{aligned}
$$

Putting $X_{3}=\xi$ in (5.1), we obtain

$$
\begin{aligned}
\left(\nabla_{W} S\right)\left(X_{2}, \xi\right) & =-2 n \alpha^{2}\left[\tilde{\mathcal{A}}_{1}(W) \eta\left(X_{2}\right)+\tilde{\mathcal{B}}_{1}\left(X_{2}\right) \eta(W)\right]+\tilde{\mathcal{D}}_{1}(\xi) S\left(X_{2}, W\right) \\
& +\tilde{\mathcal{A}}_{2}(W) \eta\left(X_{2}\right)+\tilde{\mathcal{B}}_{2}\left(X_{2}\right) \eta(W)+\tilde{\mathcal{D}}_{2}(\xi) g\left(X_{2}, W\right) .
\end{aligned}
$$

In view of (3.5) the relation (5.2) becomes

$$
\begin{aligned}
-2 n \alpha^{3} g\left(X_{2}, W\right)-\alpha S\left(X_{2}, W\right)= & -2 n \alpha^{2}\left[\tilde{\mathcal{A}}_{1}(W) \eta\left(X_{2}\right)+\tilde{\mathcal{B}}_{1}\left(X_{2}\right) \eta(W)\right] \\
& +\tilde{\mathcal{D}}_{1}(\xi) S\left(X_{2}, W\right)+\tilde{\mathcal{A}}_{2}(W) \eta\left(X_{2}\right) \\
& +\tilde{\mathcal{B}}_{2}\left(X_{2}\right) \eta(W)+\tilde{\mathcal{D}}_{2}(\xi) g\left(X_{2}, W\right) .
\end{aligned}
$$

Setting $W=X_{2}=\xi$ in (5.3) and using (2.1), (2.2) and (2.7), we get

$$
2 n \alpha^{2}\left[\tilde{\mathcal{A}}_{1}(\xi)+\tilde{\mathcal{B}}_{1}(\xi)+\tilde{\mathcal{D}}_{1}(\xi)\right]=\tilde{\mathcal{A}}_{2}(\xi)+\tilde{\mathcal{B}}_{2}(\xi)+\tilde{\mathcal{D}}_{2}(\xi) .
$$


Again, putting $W=\xi$ in (5.3), we get

$$
2 n \alpha^{2}\left[\tilde{\mathcal{A}}_{1}(\xi) \eta\left(X_{2}\right)+\tilde{\mathcal{B}}_{1}\left(X_{2}\right)+\tilde{\mathcal{D}}_{1}(\xi) \eta\left(X_{2}\right)\right]=\tilde{\mathcal{A}}_{2}(\xi) \eta\left(X_{2}\right)+\tilde{\mathcal{B}}_{2}\left(X_{2}\right)+\tilde{\mathcal{D}}_{2}(\xi) \eta\left(X_{2}\right) .
$$

Setting $X_{2}=\xi$ in (5.3) and then using (2.1), (2.2) and (2.7), we obtain

$$
2 n \alpha^{2}\left[\tilde{\mathcal{A}}_{1}(W)+\tilde{\mathcal{B}}_{1}(\xi) \eta(W)+\tilde{\mathcal{D}}_{1}(\xi) \eta(W)\right]=\tilde{\mathcal{A}}_{2}(W)+\tilde{\mathcal{B}}_{2}(\xi) \eta(W)+\tilde{\mathcal{D}}_{2}(\xi) \eta(W) .
$$

Replacing $X_{2}$ by $W$ in (5.5) and then adding the resultant with (5.6), we obtain

$$
\begin{aligned}
& 2 n\left[\alpha^{2} \tilde{\mathcal{A}}_{1}(W)+\alpha^{2} \tilde{\mathcal{B}}_{1}(W)\right]-\left[\tilde{\mathcal{A}}_{2}(W)+\tilde{\mathcal{B}}_{2}(W)\right] \\
& =-2 n\left[\alpha^{2} \tilde{\mathcal{A}}_{1}(\xi)+\alpha^{2} \tilde{\mathcal{B}}_{1}(\xi)+\alpha^{2} \tilde{\mathcal{D}}_{1}(\xi)\right] \eta(W) \\
& +\left[\tilde{\mathcal{A}}_{2}(\xi)+\tilde{\mathcal{B}}_{2}(\xi)+\tilde{\mathcal{D}}_{2}(\xi)\right] \eta(W)-2 n \alpha^{2} \tilde{\mathcal{D}}_{1}(\xi) \eta(W)+\tilde{\mathcal{D}}_{2}(\xi) \eta(W) .
\end{aligned}
$$

Due to (5.4), equation (5.7) turns into

$$
\begin{gathered}
2 n \alpha^{2}\left[\tilde{\mathcal{A}}_{1}(W)+\tilde{\mathcal{B}}_{1}(W)\right]+2 n \alpha^{2} \tilde{\mathcal{D}}_{1}(\xi) \eta(W) \\
=\left[\tilde{\mathcal{A}}_{2}(W)+\tilde{\mathcal{B}}_{2}(W)\right]+\tilde{\mathcal{D}}_{2}(\xi) \eta(W) .
\end{gathered}
$$

Then taking, $X_{2}=W=\xi$ in (5.1), we obtain

$$
\begin{gathered}
2 n \alpha^{2}\left[\tilde{\mathcal{A}}_{1}(\xi)+\tilde{\mathcal{B}}_{1}(\xi)\right] \eta\left(X_{3}\right)+2 n \alpha^{2} \tilde{\mathcal{D}}_{1}\left(X_{3}\right) \\
=\left[\tilde{\mathcal{A}}_{2}(\xi)+\tilde{\mathcal{B}}_{2}(\xi)\right] \eta\left(X_{3}\right)+\tilde{\mathcal{D}}_{2}\left(X_{3}\right) .
\end{gathered}
$$

Replacing $X_{3}$ by $W$ in (5.9) and adding with (5.8), we find out

$$
\begin{aligned}
& 2 n \alpha^{2}\left[\tilde{\mathcal{A}}_{1}(W)+\tilde{\mathcal{B}}_{1}(W)+\tilde{\mathcal{D}}_{1}(W)\right]+2 n \alpha^{2}\left[\tilde{\mathcal{A}}_{1}(\xi)+\tilde{\mathcal{B}}_{1}(\xi)+\tilde{\mathcal{D}}_{1}(\xi)\right] \eta(W) \\
& =\left[\tilde{\mathcal{A}}_{2}(W)+\tilde{\mathcal{B}}_{2}(W)+\tilde{\mathcal{D}}_{2}(W)\right]+\left[\tilde{\mathcal{A}}_{2}(\xi)+\tilde{\mathcal{B}}_{2}(\xi)+\tilde{\mathcal{D}}_{2}(\xi)\right] \eta(W) .
\end{aligned}
$$

Using equation (5.4), we get from the (5.10) equation

$$
2 n \alpha^{2}\left[\tilde{\mathcal{A}}_{1}(W)+\tilde{\mathcal{B}}_{1}(W)+\tilde{\mathcal{D}}_{1}(W)\right]=\left[\tilde{\mathcal{A}}_{2}(W)+\tilde{\mathcal{B}}_{2}(W)+\tilde{\mathcal{D}}_{2}(W)\right] .
$$

This leads to the following theorem.

Theorem 5.1. In a generalized weakly Ricci symmetric $\alpha$-cosymplectic manifold $\left(M^{2 n+1}, g\right) \quad(n \geq 1)$, the sum of the associated 1 -forms is related by (5.11).

Again from (5.3), we have

$$
\begin{aligned}
S\left(X_{2}, W\right)= & -\frac{\left[2 n \alpha^{3}+\tilde{\mathcal{D}}_{2}(\xi)\right]}{\alpha+\tilde{\mathcal{D}}_{1}(\xi)} g\left(X_{2}, W\right)+\frac{\left[2 n \alpha^{2} \tilde{\mathcal{A}}_{1}(W)-\tilde{\mathcal{A}}_{2}(W)\right]}{\alpha+\tilde{\mathcal{D}}_{1}(\xi)} \eta\left(X_{2}\right) \\
& +\frac{\left[2 n \alpha^{2} \tilde{\mathcal{B}}_{1}\left(X_{2}\right)-\tilde{\mathcal{B}}_{2}\left(X_{2}\right)\right]}{\alpha+\tilde{\mathcal{D}}_{1}(\xi)} \eta(W) .
\end{aligned}
$$

From (5.6), we get

$$
2 n \alpha^{2} \tilde{\mathcal{A}}_{1}(W)-\tilde{\mathcal{A}}_{2}(W)=\left[-2 n \alpha^{2}\left(\tilde{\mathcal{B}}_{1}(\xi)+\tilde{\mathcal{D}}_{1}(\xi)\right)+\left(\tilde{\mathcal{B}}_{2}(\xi)+\tilde{\mathcal{D}}_{2}(\xi)\right)\right] \eta(W) .
$$

Using (5.4) in (5.5), we obtain

$$
\left[2 n \alpha^{2} \tilde{\mathcal{B}}_{1}(\xi)-\tilde{\mathcal{B}}_{2}(\xi)\right] \eta\left(X_{2}\right)=2 n \alpha^{2} \tilde{\mathcal{B}}_{1}\left(X_{2}\right)-\tilde{\mathcal{B}}_{2}\left(X_{2}\right) .
$$

In view of (5.12), (5.13) and (5.14), we have

$$
S\left(X_{2}, W\right)=-\frac{\left[2 n \alpha^{3}+\tilde{\mathcal{D}}_{2}(\xi)\right]}{\alpha+\tilde{\mathcal{D}}_{1}(\xi)} g\left(X_{2}, W\right)+\frac{\left[-2 n \alpha^{2} \tilde{\mathcal{D}}_{1}(\xi)+\tilde{\mathcal{D}}_{2}(\xi)\right]}{\alpha+\tilde{\mathcal{D}}_{1}(\xi)} \eta\left(X_{2}\right) \eta(W) .
$$

This leads to the following theorems.

Theorem 5.2. A generalized weakly Ricci symmetric $\alpha$-cosymplectic manifold is an $\eta$ Einstein space provided $\tilde{\mathcal{D}}_{1}(\xi) \neq-\alpha$ 
Theorem 5.3. In an $\alpha$-cosymplectic manifold the following table is hold.

\begin{tabular}{ll}
\hline Type of curvature restriction & $\begin{array}{l}\text { Nature of the space corresponding to } \\
\text { curvature restriction }\end{array}$ \\
\hline locally symmetric space & Einstein space \\
\hline locally recurrent space & $\eta$ - Einstein space \\
\hline generalized recurrent space & $\eta$ - Einstein space \\
\hline pseudo symmetric space & $\eta$-Einstein space \\
\hline generalized pseudo symmetric space & $\eta$ - Einstein space \\
\hline semi-pseudo symmetric space & $\eta$ - Einstein space \\
\hline generalized semi-pseudo symmetric space & $\eta$-Einstein space \\
\hline almost pseudo symmetric space & $\eta$-Einstein space \\
\hline almost generalized pseudo symmetric space & $\eta$-Einstein space \\
\hline weakly symmetric space & $\eta$-Einstein space \\
\hline
\end{tabular}

\section{References}

[1] N. Aktan, M. Yıldırım and C. Murathan, Almost f-cosymplectic manifolds, Mediterr. J. Math., 11 (2), 775-787, 2014.

[2] M.A. Akyol, Conformal anti-invariant submersions from cosymplectic manifolds, Hacet. J. Math. Stat., 46 (2), 176-192, 2017.

[3] K.K. Baishya and P.R. Chowdhury, On Generalized weakly symmetric Kenmotsu manifolds, Bol. Soc. Paran. Mat., 39 (6), 211-222, 2021.

[4] S. Beyendi, G. Ayar and N. Aktan, On a type of $\alpha$-cosymplectic manifolds, Commun. Fac. Sci. Univ. Ank. Ser. A1 Math. Stat. 68 (1), 852-861, 2019.

[5] D.E. Blair, Contact Manifolds in Riemannian Geometry, Lect. Notes Math. 509, Springer-Verlag, Berlin, 1976.

[6] E. Cartan, Sur une classes remarquable d'espaces de Riemannian, Bull. Soc. Math. France, 54, 214-264, 1926.

[7] M.C. Chaki, On pseudo Ricci symmetric manifolds, Bulg. J. Physics, 15 (6), 526-531, 1988.

[8] U.C. De and S. Bandyopadhyay, On weakly symmetric spaces, Acta Math. Hung., 87(3), 205-212, 2000.

[9] R.S.D. Dubey, Generalized recurrent spaces, Indian J. Pure Appl. Math., 10 (12), 1508-1513, 1979.

[10] Y. Gündüzalp and M.A. Akyol, Conformal slant submersions from cosymplectic manifolds, Turk. J. Math., 42 (5), 2672-2689, 2018.

[11] S.K. Hui, A.A. Shaikh and I. Roy, On totaly umbilical hypersurfaces of weakly conharmonically symmetric spaces, Global journal of Pure and Applied Mathematics, 10 (4), 28-31, 2010.

[12] S.K. Jana and A.A. Shaikh, On quasi-conformally flat weakly Ricci symmetric manifolds, Acta Math. Hung., 115 (3), 197-214, 2007.

[13] T.W. Kim and H.K. Pak, Canonical foliations of certain classes of almost contact metric structures, Acta Math, Sinica, Eng. Ser. Aug., 21 (4), 841-846, 2005.

[14] F. Özen and S. Altay, On weakly and pseudo symmetric Riemannian spaces, Indian J. Pure Appl. Math., 33 (10), 1477-1488, 2001.

[15] H. Öztürk, C. Murathan, N. Aktan and A.T. Vanli, Almost $\alpha$-cosymplectic $f$ manifolds, An. Stiint. Univ. Al. I. Cuza Iasi. Mat.(NS), 60 (1), 211-226, 2014.

[16] M. Prvanovic, On weakly symmetric Riemannian manifolds, Pub. Math. Debrecen, 46, 19-25, 1995.

[17] A.A. Shaikh and K.K.Baishya, On weakly quasi-conformally symmetric manifolds, Soochow Journal of Mathematics 31 (4), 581-595, 2005. 
[18] L. Tamassy and T.Q. Binh, On weakly symmetric and weakly projective symmetric Riemannian manifolds, Coll. Math. Soc., J. Bolyai, 56, 663-670, 1989.

[19] M. Tarafdar and M.A.A. Jawarneh, Semi-Pseudo Ricci Symmetric manifold, J. Indian Inst. Sci., 73, 591-596, 1993.

[20] A.G. Walker, On Ruses space of recurrent curvature, Proc. London Math. Soc., 52, 36-54, 1950.

[21] M. Yıldırım and S. Beyendi, On almost generalized weakly symmetric $\alpha$-cosymplectic manifolds, Univers. J. Math. Appl., 3 (4), 156-159, 2020. 